\title{
Diluted magnetic layered semiconductor InSe:Mn with high Curie temperature
}

\author{
G.V. Lashkarev ${ }^{1}$, V.I. Sichkovskyi ${ }^{1}$, M.V. Radchenko ${ }^{1}$, P. Aleshkevych ${ }^{2}$, O.I. Dmitriev ${ }^{1}$, P.E. Butorin ${ }^{1,3}$, \\ Z.D. Kovalyuk ${ }^{1}$, R. Szymczak', A. Slawska-Waniewska², N. Nedelko², R. Yakiela ${ }^{2}$, A.M. Balagurov ${ }^{3}$, \\ A.I. Beskrovnyy ${ }^{3}$, W. Dobrowolski ${ }^{2}$ \\ ${ }^{1}$ I.M. Frantsevich Institute for Problems of Materials Science, \\ National Academy of Sciences of Ukraine, 3, Krzhizhanivskogo str., 03680 Kyiv, Ukraine \\ ${ }^{2}$ Institute of Physics, Polish Academy of Sciences, Al. Lotnikow 32/46, 02-668 Warsaw, Poland \\ ${ }^{3}$ Joint Institute for Nuclear Research, Joliot-Curie 6, 141980 Dubna, Russia
}

\begin{abstract}
We present a detailed study of layered semiconductor InSe doped with Mn. Xray and neutron diffraction analyses of (In,Mn)Se single crystals show the presence of a main phase as $\operatorname{In}_{1-\chi} \mathrm{Mn}_{\chi}$ Se solid solution, the second antiferromagnetic MnSe phase, and traces of $\mathrm{In}_{4} \mathrm{Se}_{3}$. Magnetic measurements reveal ferromagnetic behavior of (In,Mn)Se with the Curie temperature about $800 \mathrm{~K}$. The ferromagnetic cluster model and exchange interaction via 2D electron gas, as the reasons of spontaneous magnetization, are discussed. The dramatic transformation of (In,Mn)Se electron spin resonance (ESR) spectra as a function of temperature is revealed. At the magnetic field perpendicular to crystallographic $c$ axis, a low-field line within the temperature range 70 down to $4.7 \mathrm{~K}$ is observed. It shifts to smaller magnetic fields with temperature decrease. Neutron diffraction studies reveal the strong rise for one of the reflection peaks with temperature decrease in the same temperature region where ESR spectra transformation occurs. This peak corresponds to double MnSe interplanar distance in the [111] direction what is a period of its magnetic lattice. Magnetic structure of (In,Mn)Se single crystal is discussed.
\end{abstract}

Keywords: diluted magnetic semiconductors, magnetic susceptibility, ESR spectrum.

Manuscript received 08.02.11; accepted for publication 14.09.11; published online 21.09.11.

\section{Introduction}

Nowadays, diluted magnetic semiconductors (DMSs) attract much attention. Dietl and Ohno [1] predicted the Curie point higher than the room temperature for $\mathrm{ZnO}$ and GaN doped with manganese at a high hole concentration. After this publication, the interest to the problem of the ferromagnetic (FM) ordering in semiconductors at temperatures higher $300 \mathrm{~K}$ and to application of DMS in spintronic devices has largely grown.

Unfortunately, the achievement of a high Curie temperature in spintronic semiconducting materials appeared to be a very difficult task. DMSs have the following lacks as: (1) low solubility of TMs in semiconductor hosts for formation of FM ordering; (2) a tendency of TMs to form other FM phases as inclusions, which simulates the FM state of DMSs (phantom of ferromagnetism) [2]; (3) an inhomogeneous distribution of magnetic ions in the host, leading in some cases to spatial localization of current carriers and to a hopping mechanism of conduction; (4) low mobility $\left(\leq 10 \mathrm{~cm}^{2} \mathrm{~V}^{-1} \mathrm{~s}^{-1}\right)$ of current carriers as caused by scattering on magnetic impurities and holes.

Layered crystals, belonging to the group of III-VI compounds and characterized by high anisotropy of chemical bonds (strong ionic-covalent bond within the plane of crystal layers and weak Van der Waals one 
between the layers) attract a particular attention. InSe is the only crystal in this group, in which sufficiently high electron or hole concentrations can be achieved. Bond anisotropy induces two-dimensional (2D) electron conductance at temperatures below the critical one. Current carriers with the average Hall concentration of about $10^{12}-10^{14} \mathrm{~cm}^{-3}$ in the crystal are accumulated in 2D regions of ionic-covalent layers near the Van der Waals gap, thus leading to the surface concentration of electrons about $10^{11} \mathrm{~cm}^{-2}$, which provides an opportunity for the Shubnikov-de Haas effect to be observed [3]. In addition, realization of magnetic interactions depends on the ability of layered crystals to redistribute impurities in their bulk, when they are accumulated in the Van der Waals gap, forming 2D aggregates or lattices [4].

Layered III-VI DMSs studied up to date by various authors revealed unusual magnetic properties. In $\mathrm{Ga}_{1-x} \mathrm{Mn}_{x} \mathrm{Se} \quad(x=0.012)$, a broad magnetization peak between 120 and $195 \mathrm{~K}$ with an apex at $160 \mathrm{~K}$ and a sharp change of magnetization at $119 \mathrm{~K}$ were observed; the peak being broadened and shifted to lower temperatures with a magnetic field increase [5]. This phenomenon was associated with a short-range antiferromagnetic ordering. It was established that $\mathrm{Ga}_{1-x} \mathrm{Mn}_{x} \mathrm{Se}(x=0.011, x=0.066)$ obeys the CurieWeiss law within the interval 50 to $235 \mathrm{~K}$. But, at $T=10.9 \mathrm{~K}$, a sharp maximum of magnetization was observed, which the authors considered as a spin glass state and suggested the presence of Mn-S-Mn pairs in the crystal [6]. Pekarek et al. [7] revealed that magnetic behavior of $\mathrm{Ga}_{1-x} \mathrm{Fe}_{x} \mathrm{Se}(x=0.05)$ is consistent with Van Vleck paramagnetism over the temperature range $5-400 \mathrm{~K}$. Investigation of $\operatorname{In}_{1-x} \mathrm{Mn}_{x} \mathrm{~S} \quad(x=0.02)$ displayed the behavior of the Curie-Weiss type and spin glass state below $10 \mathrm{~K}[8,9]$. The temperature hysteresis of magnetization in the interval $90-290 \mathrm{~K}$ was observed for $\operatorname{In}_{1-x} \mathrm{Mn}_{x} \mathrm{Se}$ crystals $(x=0.01, x=0.10) \quad$ [10]. Below and above the hysteresis region, the paramagnetic signal obeyed the Curie-Weiss law with a negative Curie-Weiss temperature $J_{\text {eff }} / k_{\mathrm{B}}=-240 \mathrm{~K}$ that indicated antiferromagnetic interaction between $\mathrm{Mn}$ ions. Two magnetic subsystems were detected in unannealed $\mathrm{In}_{1-x} \mathrm{Mn}_{x} \mathrm{Se}(x=0.0125)$ sample $[11,12]$. The authors supposed that $\mathrm{Mn}$ ions are located both in the ioniccovalent layers and in interlayer Van der Waals space simultaneously and redistribute to the latter during sample annealing. The hysteresis loops with a weak coercive field at $T<50 \mathrm{~K}$ were observed. Thus, the considered experimental data demonstrate a large divergence in behavior of III-VI diluted magnetic layered crystals and difficulties in the revealing their general regularities. Besides, Pekarek et al. [13] reported that $\mathrm{In}_{1-\chi} \mathrm{Mn}_{x} \mathrm{Se}$ samples were cut from an ingot grown by Bridgman method supposing that composition of the samples have the same nominal Mn concentration as was put into the charge. Nevertheless, Slyn'ko et al. [11, 12] noticed non-uniform distribution of $\mathrm{Mn}$ in the ingot. Instead of initial composition in the melt about 1 at.\% they registered 0.15 at. $\%$ at the beginning of the ingot, while near its end $\mathrm{Mn}$ concentration was as high as 3.6 at.\%. Therefore, Mn concentration in the charge does not correspond to the real $\mathrm{Mn}$ content in samples cut out of different parts of this ingot.

It is necessary to underline that the information about phase and real element composition of III-VI DMSs is absent in the cited papers [5-13]. Nevertheless, this knowledge is of great importance for regular discussion of the results.

The further investigations of (In,Mn)Se crystals [14] explained some of their previously unclear properties such as nature of the magnetic susceptibility peaks at $\sim 163$ and $266 \mathrm{~K}$. The ferromagnetic ordering with the Curie temperature $T_{C}>350 \mathrm{~K}$ was revealed [15]. There appeared strong motivation to continue the study of this magnetic semiconductor. Therefore, Mndoped InSe is an interesting unusual DMS.

In this paper, the magnetization, electron spin resonance, and neutron diffraction spectra of ( $\mathrm{In}, \mathrm{Mn}) \mathrm{Se}$ crystals in a wide range of temperatures and magnetic fields were investigated. The phase and element composition of samples were thoroughly studied.

\section{Experimental details}

InSe single crystals doped with manganese were grown using the Bridgman method. The solubility limit of Mn in III-VI crystals is unknown till now. The coefficient of impurity distribution is also unknown but seems extremely low because impurity is intensively pushed out from the solid phase to liquid one [11]. Therefore, the definite content of $\mathrm{Mn}$ in charge $C$ is not uniformly distributed along the single crystal: at the beginning of the ingot the content of $\mathrm{Mn} C_{b}<<C$, while at the end of the ingot one observes $C_{e}>>C$. Thus it is necessary to define real content of $\mathrm{Mn}$ in each studied sample.

The Mn content in the specimens determined by the $\mathrm{X}$-ray fluorescent analysis was equal 5 at.\% and 0.4 at. $\%$ (mentioned hereinafter, respectively, as type I and type II). Samples were annealed at the temperature of $590 \mathrm{~K}$ for 70 hours (type I) and for 140 hours (type II). $\mathrm{X}$-ray diffraction (XRD) technique showed that the hexagonal phase of $\mathrm{InSe}(P 63 / \mathrm{mmc})$ with the lattice periods $a=4.0026 \AA$ and $c=16.634 \AA$ was the main phase ( $>90$ vol. $\%$ of the crystal). In addition, the specimens contained inclusions ( $<10$ vol.\%) of a second (cubic at room temperature) $\mathrm{MnSe}$ phase (Fm3m, $a=5.456 \AA$ ). This estimation of composition was carried out by comparison the intensities of X-ray reflections and is a rough one.

In the low temperature interval 2.4-300 K magnetic properties of the material were studied using SQUID magnetometer, Quantum Design MPMS-5. High temperature dc magnetization measurements over the temperature range $300-850 \mathrm{~K}$ were performed by the 
vibrating sample magnetometer (VSM) Oxford Instruments Ltd. The sample was fixed onto ceramic holder by the Autostic high performance ceramic industrial cement FC8

The electron spin resonance (ESR) spectra were researched in the interval $4.4-300 \mathrm{~K}$ using the EMX Bruker ER083CS spectrometer operating at a fixed frequency of $9.38 \mathrm{GHz}$ in the X-band.

Neutron diffraction (ND) experiments were performed with the diffractometer DN-2 in the Frank Neutron Physics Laboratory of Joint Institute for Nuclear Researches, Dubna, using neutron beam of the IBR-2 pulsed reactor. DN-2 is the time-of-flight neutron spectrometer utilizing the $2 \mathrm{D}$ position-sensitive detector for data acquisition. The diffraction patterns were measured at several scattering angles and sample orientations for revealing peculiarities in the wavelength dependence of diffraction intensities and texture effects.

\section{Results and discussion}

\subsection{Magnetic moment}

The temperature dependence of the magnetic moment (MM) of (In,Mn)Se of type I sample within the range $2.4-850 \mathrm{~K}$ is shown in Fig. 1. The good correspondence of experimental data for two methods (SQUID and VSM) was observed up to $550 \mathrm{~K}$. The sample heated up to $850 \mathrm{~K}$ and then cooled down does not display the same properties as before the high temperature experiment. The measurements were carried out in magnetic field $6 \mathrm{kOe}$. In the case of SQUID researches, the sample was preliminary cooled in zero magnetic field to the minimal temperature (zero-field-cooled regime). Three peaks were registered at temperatures 150,275 , and $670 \mathrm{~K}$. Below $77 \mathrm{~K}$, the MS grows up to the minimal temperature of measurements $(2.4 \mathrm{~K})$.

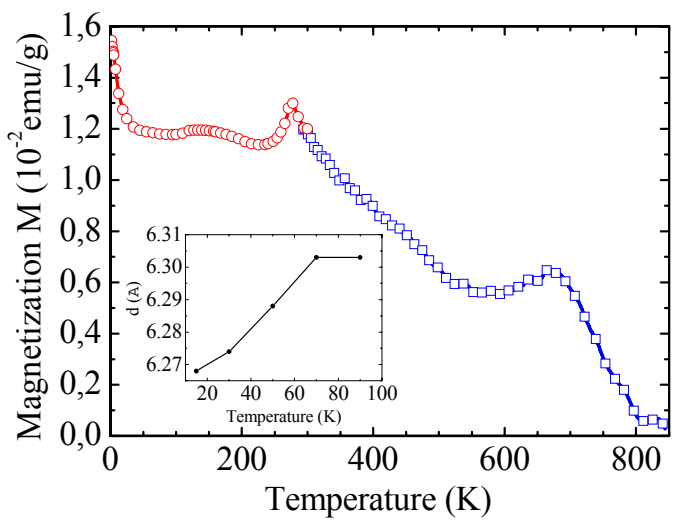

Fig. 1. Temperature dependence of the magnetic moment of (In,Mn)Se sample of type I. The dependences were measured by a SQUID magnetometer within the temperature range 2.4 to $300 \mathrm{~K}$ (circles) and by a vibrating magnetometer at temperature increase over the range $300-845 \mathrm{~K}$ (squares). The inset shows the temperature dependence of the magnetic cell parameter $d_{\frac{1}{2} \frac{1}{2} \frac{1}{2}}$ for MnSe.

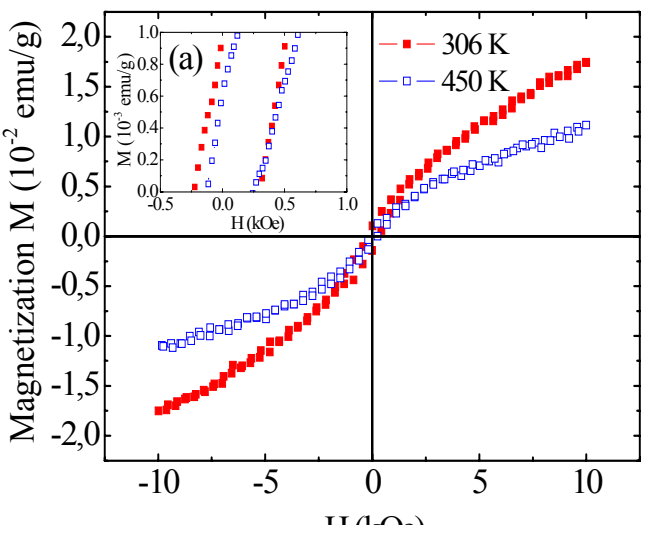

Fig. 2. Hysteresis loops as a function of magnetic field for (In,Mn)Se sample of type I at various temperatures. Inset: (a) the enlarged middle part of the loops with the coercivity.

The magnetization dependences on the magnetic field $M(H)$ were studied by VSM at several temperatures within $306-450 \mathrm{~K}$. Some of the magnetic hysteresis 1oops are depicted in Fig. 2. The inset (a) shows the magnified middle part of the loops.

\subsection{ESR spectra}

Studies of ESR spectra revealed their transformation in samples of type II at temperature decrease from 270 down to $4.7 \mathrm{~K}$ (Fig. 3). Within the temperature range $70-21 \mathrm{~K}$, the low-field line appeared simultaneously with the decay of the main line that corresponds to $\mathrm{Mn}^{2+}$ ions with a $g$-factor value $\approx 2$. The low-field line increases its amplitude and shifts towards smaller magnetic fields with lowering the temperature.

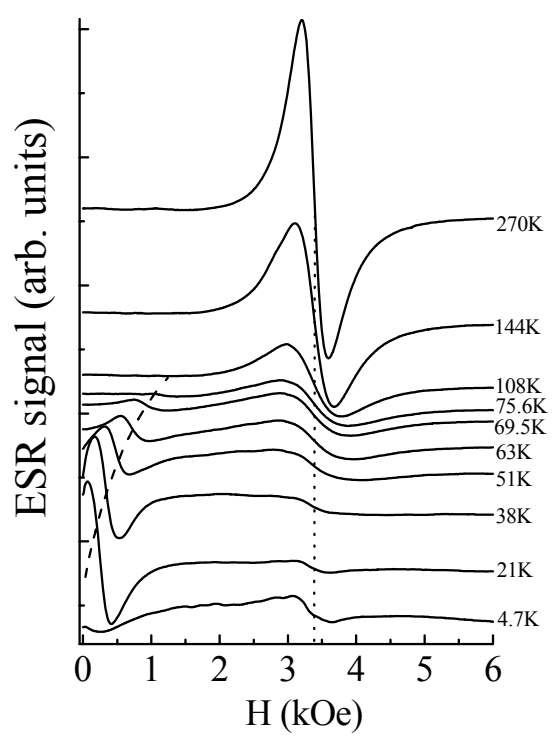

Fig. 3. ESR spectra of (In,Mn)Se of type II at various temperatures for $\mathbf{H} \perp c$ axis. The dashed line shows the shift of resonant magnetic field for low-field line with temperature decrease. The dotted line indicates the resonant magnetic field for line with the $g$-factor value $\approx 2$. 
ESR anisotropy at $300 \mathrm{~K}$ is absent in (In,Mn)Se crystals. The line shape at magnetic field $\mathbf{H}$ parallel to $C$ axis (normal to layer plane) and $\mathbf{H}$ perpendicular to $c$ axis (in layer plane) are the same. Nevertheless, at lower temperatures the anisotropic low-field ESR line appeared (Fig. 4). At $T=50 \mathrm{~K}$ the resonant field decreases, and the line intensity increases at magnetic field rotation between directions from $\mathbf{H} \| c$ to $\mathbf{H} \perp c$.

For a comparison, we studied the ESR spectra of $\mathrm{Mn}$-free InSe. The ESR signal was not observed within $100-300 \mathrm{~K}$. At lower temperatures, the strong absorption of the unknown nature was registered.

\subsection{Neutron diffraction researches}

From ND patterns, the additional data about phase state (structural and magnetic) of (In,Mn)Se were obtained. For (In,Mn)Se sample of type I, the deterioration of crystal lattice prevents sliding of crystal layers and observation of ND. Opposite to it, for the type II sample by ND studies it was found that beside the main hexagonal phase there are diffraction peaks belonging to the MnSe phase and to the small amount of $\mathrm{In}_{4} \mathrm{Se}_{3}$ phase.

To study the effects of magnetic ordering, experiments at various temperatures were carried out. The peak corresponding to $d=6.275 \AA$ was observed, which does not belong to the InSe structure. Its intensity $I$ is nearly doubled, if temperature decreases from 50 down to $10 \mathrm{~K}$, while the increase in intensities of the neighboring peaks $d_{004}=4.157 \AA$ and $d_{002}=8.313 \AA$ is much less.

The analysis shows that this diffraction peak $d=6.275 \AA$ is unresolved superposition of these two components: at $d=6.154 \AA$ and $d=6.303 \AA$. The first (weakly dependent on temperature) belongs to the $\operatorname{In}_{4} \mathrm{Se}_{3}$ phase. The intensity of the second component strongly depends on temperature (Table I) and can be attributed to the MnSe magnetic diffraction peak $d_{111}$.

Extrapolating $I(T)$ dependence to zero intensity, one can find the phase-transition temperature as $T=(70 \pm 10) \mathrm{K}$.

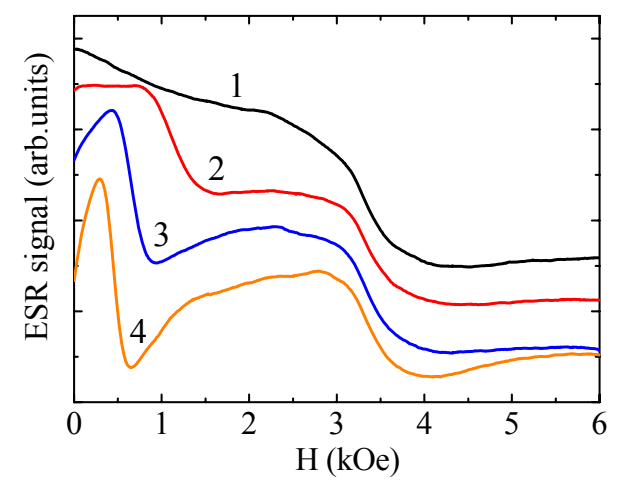

Fig. 4. ESR spectra of (In,Mn)Se of type II at $T=50 \mathrm{~K}$. The arrows indicate the ESR spectrum at various orientations of the magnetic field $\mathbf{H}$ to crystallographic $c$ axis: $\mathbf{H}$ parallel to $c$ axis (1), $\mathbf{H}$ perpendicular to $c$ axis (4), and intermediate positions (2 and 3$)$.

\subsection{Discussion of the results}

Doping the layered InSe crystal with manganese forms a complicated magnetic structure. During crystal growth, manganese distributes inside the layers and in the interlayer spaces. One can suppose that a prolonged annealing should result, on the one hand, in a more uniform doping of the ionic-covalent Se-In-In-Se layers owing to the statistical substitution of In sites by Mn. On the other hand, Mn shifts to the interlayer space and concentrates near defects of the crystal structure as well.

According to the results of XRD studies [14], the crystal lattice periods are smaller in the (In,Mn)Se sample of type I $(a=4.0026 \AA$ and $c=16.634 \AA)$ as compared with those in the undoped $\operatorname{InSe}(a=4.005 \AA$ and $c=16.64 \AA$ ) [16]. The variation of the lattice periods evidences for the formation of the main phase - solid solution $\operatorname{In}_{1-x} \mathrm{Mn}_{x} \mathrm{Se}$, where $\mathrm{Mn}$ ions substitute In ones.

Assuming that the manganese impurity is distributed statistically over the crystal, its concentration $N$ is $8.6 \times 10^{20} \mathrm{~cm}^{-3}$ for 5.0 at. $\%$, and the distance between ions $R=N^{-1 / 3}$ is approximately equal to $23 \AA$. The activation of the ordinary Ruderman-Kittel-Kasuya-Yosida (RKKY) mechanism of indirect exchange interaction between magnetic ions is impossible, because the average concentration of free charge carriers is low $\left(\sim \sim 10^{14} \mathrm{~cm}^{-3}\right)$. In this case, the hypothesis of Slyn'ko et al. $[11,12]$ that the ferromagnetic order exists only in restricted regions of the crystal or in clusters, remains the most natural one. The low solubility of TM in InSe and the formation of structural defects are the additional origin of the nonuniform distribution of manganese in this crystal. These obstacles can favor formation of magnetically ordered clusters owing to the superexchange between Mn ions via anions (Se) [17]. The exchange interaction in such clusters can occur in Mn-Se-Mn pairs and, probably, in Mn-In-SeMn ones [5]. Ferromagnetism was confirmed by the existence of hysteresis loops of the magnetic moment at least up to $450 \mathrm{~K}$. The magnitudes of coercivity and magnetization of saturation have the same order as in the case of SQUID measurements for appropriate temperatures [15]. The Curie temperature $T_{\mathrm{C}}$ of ferromagnetically ordered clusters is approximately $800 \mathrm{~K}$ (Fig. 1).

We can also suppose that the ferromagnetic state arises owing to the exchange interaction between $\mathrm{Mn}$ ions via the $2 \mathrm{D}$ electron gas located at the interface between the Se-In-In-Se layer and the Van der Waals gap (2D ferromagnetism). Nevertheless, the theoretical considerations of this mechanism were carried out only for quantum wells at very low temperatures [18].

An estimation of magnetic moment per $\mathrm{Mn}$ ion gives the extremely low magnitude $5 \times 10^{-3} \mu_{\mathrm{B}}$. It means that only a small part of manganese participate in FM ordering. Its larger part forms AFM MnSe phase and antiferromagnetically coupled Mn-Mn pairs.

The features of the chemical bond in (In,Mn)Se can lead to the generation of $\mathrm{Mn}^{3+}\left(d^{4}\right)$ and $\mathrm{Mn}^{+}\left(d^{6}\right)$ ions, which is characterized by Van Vleck behavior due to even 
number of $d$-electrons [17]. Such ions can be responsible for the temperature-independent magnetic moment.

In previous studies $[14,15]$, we investigated the temperature dependence of the magnetic susceptibility for InSe sample containing 5 at. $\% \mathrm{Mn}$ in the interval $2.4-300 \mathrm{~K}$ in the zero-field-cooled regime. Pronounced peaks at 163 and $266 \mathrm{~K}$ were attributed to antiferromagnetic transformations of the MnSe second phase $\left(T_{N 1}=130 \mathrm{~K}, T_{N 2}=266 \mathrm{~K}\right)$ [19]. It should be noted that magnetic properties of $\mathrm{MnSe}$ in the bulk form are not clear. The situation is even more complex in nanostructured $\mathrm{MnSe}$ where the lattice distortions and surface/interfacial layers are expected to favor the uncompensated anitiferromagnetic structure with a net magnetic moment. Thus, the ferromagnetic-like behavior and coercivity observed in (In,Mn)Se sample can also originate from the boundary regions of the layered $\mathrm{MnSe}$ inclusions segregated during annealing of crystal.

The nature of the third peak of $\mathrm{MM}$ at $670 \mathrm{~K}$ (Fig. 1) can be due to the transformation of the material that occurs at temperatures above the annealing temperature of the sample. This transformation can be related with transition of unstable $\beta-\mathrm{MnSe}$ to $\alpha-\mathrm{MnSe}$ phase that is known to take place at $625 \mathrm{~K} \mathrm{[20]} \mathrm{and} \mathrm{also}$ with the further segregation of Mn.

The exchange interaction between manganese ions in the crystal and the nonequivalence of their local symmetry result in the absence of the usual group of six lines of hyperfine splitting (characteristic to diluted $\mathrm{Mn}^{2+}$ ions) in the ESR spectrum of InSe. These lines broaden due to dipole-dipole interaction that leads to the disappearance of hyperfine structure. Simultaneously, the line with $g \approx 2$ can include the line of ferromagnetic resonance in the ordered main phase $\mathrm{In}_{1-x} \mathrm{Mn}_{x} \mathrm{Se}$. The low-field ESR line (Fig. 3) appears in the temperature range where magnetic moment of $\mathrm{MnSe}$ phase rises at temperature lowering below $70 \mathrm{~K}$ along with attenuation of the mentioned strong line $(g \approx 2)$. It testifies to magnetic interaction of FM clusters with MnSe layers. We suppose that low resonance field is due to internal magnetic field of FM ordered MnSe phase at $T<70 \mathrm{~K}$. This field mediated by unusual FM state of MnSe phase in its plane adds to the external field of ESR spectrometer increasing the total magnetic field in which low-field resonance is observed. The ferromagnetic state of MnSe layers at $T<70 \mathrm{~K}$ was proved by revealing the magnetic sublattice of $\mathrm{MnSe}$ at these temperatures.

The sharp increase of MM (In,Mn)Se and MnSe as a function of temperature below $70 \mathrm{~K}$ (Fig. 1), which does not disappear in strong magnetic field $(H=6 \mathrm{kOe})$ can be attributed to phase transformation with significant decrease of the magnetic lattice period for MnSe plane inclusions. This conclusion is confirmed by the temperature dependence of the magnetic cell parameter $d_{111}$ below

$$
\overline{2} \frac{1}{2} \frac{1}{2}
$$

$70 \mathrm{~K}$ obtained from the ND spectra and displayed in the inset of Fig. 1. Therefore, in the low temperature region the magnetic transformation of ferromagnetic type in $\mathrm{MnSe}$ plane inclusions occurs. As in the case of MnTe crystals, the magnetic cell of MnSe could be viewed as Mn moments aligned ferromagnetically in the basal planes and these planes stack antiferromagnetically along the $c$ axis which is in agreement with those reported by Efrem et al. [21]. Owing to the magnetic structure of MnSe, the magnetic interactions seem to be strongly associated with the structural parameters and any change in the latter would affect the overall magnetic interactions. Below $70 \mathrm{~K}$, the magnetic lattice parameter decreases sharply with diminishing temperature due to a decrease of $c$ and $a$ values. The Mn-Mn shortest interatomic distance in the basal plane is equal to $a$. Therefore, the decrease in $a$ would strengthen the ferromagnetic interactions between the $\mathrm{Mn}$ ions in the basal plane. As a sharp increase in the susceptibility is observed both for $\mathrm{Mn}(\mathrm{As}, \mathrm{Te}, \mathrm{S}, \mathrm{Sb}, \mathrm{Se})$ [21], on the one hand, and for MnSe phase in a InSe matrix, on the other hand, it is possible to assume that it is their general property.

Inclusions of the MnSe phase in the layered structure of the (In,Mn)Se matrix are rather regular: the [111] direction of the inclusions coincides with the matrix $c$ axis [001]. The thickness of each layer is larger than the size of the coherence region for X-ray radiation. The latter is equal about ten of MnSe lattice periods. This permitted us to observe the MnSe phase in X-ray and neutron diffraction spectra. Estimations of $\mathrm{MnSe}$ content in (In,Mn)Se made by a comparison of (In,Mn)Se and MnSe [19] magnetic moment shows that the content of MnSe phase is much smaller (about $0.15 \mathrm{wt} . \%$ ) than it issues from XRD analysis of (In,Mn)Se [14]. Therefore, MnSe layer inclusions are thin ones and can give its contribution to uncompensated antiferromagnetism due to an odd number of magnetically ordered planes of MnSe. Thus, the (In,Mn)Se single crystal can be considered as a selfassembled artificial layered structure composed of alternating ferromagnetic $\mathrm{In}_{1-\chi} \mathrm{Mn}_{x} \mathrm{Se}$ and antiferromagnetic MnSe layers. The similar case of alternation for intercalated and nonintercalated layers was previously observed in InSe crystals [22]. It must result in periodical change of the manganese content in the sample cross direction. This conclusion was confirmed by SIMS investigations of $\mathrm{Mn}$ depth distribution [15]. In the case of (In,Mn)Se of type II sample, we have observed well pronounced oscillations of $\mathrm{Mn}$ content. The periods of oscillations are within the range 40 to $85 \mathrm{~nm}$ [15].

Hence, the magnetic structure of the crystal is diverse, being controlled by superposition of various magnetic states, namely: ferromagnetic clusters with Curie temperature $\sim 800 \mathrm{~K}$, antiferromagnetic MnSe thin planar inclusions revealing weak FM properties below $70 \mathrm{~K}$, uncompensated antiferromagnetism of the $\mathrm{MnSe}$ layers, antiferromagnetically coupled $\mathrm{Mn}^{2+}$ pairs, and perhaps Van Vleck ions.

\section{Conclusions}

The magnetic structure of layered InSe semiconductor single crystals doped with manganese has been studied. It 
consists of the solid solution $\operatorname{In}_{1-\chi} \mathrm{Mn}_{x} \mathrm{Se}$, ferromagnetically ordered clusters with $T_{\mathrm{C}} \approx 800 \mathrm{~K}$, and the plane inclusions of the antiferromagnetic MnSe phase. The nonmonotonic temperature dependence of magnetic moment for (In,Mn)Se is caused by contribution of the MnSe phase. The existence of MnSe neutron reflections indicates that the thickness of its inclusions is larger than ten MnSe lattice periods. A decrease in the magnetic sublattice period of MnSe inclusions as a function of temperature below $70 \mathrm{~K}$ was established. This results in strengthening the ferromagnetic interactions between the $\mathrm{Mn}$ ions in the basal plane, which causes a sharp rise of the magnetic moment. The unusual temperature behavior of ESR spectra due to magnetic transformation of $\mathrm{MnSe}$ phase was observed.

\section{Acknowledgements}

The authors are grateful to V.V. Slyn'ko for her interest to the work and useful discussions, to R. Minikaev for XRD analysis of samples, and to M. Baran for help in experiment. This work was supported in part by the Program of the NAS of Ukraine "Nanosystems, nanomaterials, nanotechnologies" Grant No. 85/07-H.

\section{References}

1. T. Dietl, H. Ohno, Ferromagnetism in III-V and IIVI semiconductor structures // Physica E (Amsterdam), 9, p. 185 (2001).

2. A. Bonanni, In: Abstract book of the XXXVIII Intern. School and Confer. on Physics of Semiconductors “Jaszowiec 2009”, Krynica-Zdrój, Poland, 2009, p. 80.

3. N.B. Brandt, V.A. Kulbachinskii, G.V. Lashkarev, Z.D. Kovalyuk // Fizika tekhnika poluprovodn. 21, p. 1230 (1987), in Russian.

4. K.D. Tovstyuk, Semiconductor Materials Science. Naukova dumka, Kyiv, 1984 (in Russian).

5. T.M. Pekarek, B.C. Crooker, I. Miotkowski, A.K. Ramdas, Magnetic measurements on the IIIVI diluted magnetic semiconductor $\mathrm{Ga}_{1-x} \mathrm{Mn}_{x} \mathrm{Se} / /$ J. Appl. Phys. 83, p. 6557 (1998).

6. T.M. Pekarek, M. Duffy, J. Garner, B.C. Crooker, I. Miotkowski, A.K. Ramdas, Magnetic measurements on the layered III-VI diluted magnetic semiconductor $\mathrm{Ga}_{1-\mathrm{x}} \mathrm{Mn}_{\mathrm{x}} \mathrm{Se} / / \mathrm{J}$. Appl. Phys. 87, p. 6448 (2000).

7. T.M. Pekarek, C.L. Fuller, J. Garner, B.C. Crooker, I. Miotkowski, A.K. Ramdas, Magnetic measurements on the layered III-VI diluted magnetic semiconductor $\mathrm{Ga}_{1-x} \mathrm{Fe}_{x} \mathrm{Se} / / \mathrm{J}$. Appl. Phys. 89, p. 7030 (2001).

8. G. Franzese, Ashlee Byrd, J.L. Tracy, J. Garner, T.M. Pekarek, I. Miotkowski, A.K. Ramdas, The singlet model of the anisotropic magnetization of the III-VI diluted magnetic semiconductor $\mathrm{In}_{1-x} \mathrm{Mn}_{x} \mathrm{~S} / /$ J. Appl. Phys. 97, 10D308 (2005).
9. J.L. Tracy, G. Franzese, Ashlee Byrd, J. Garner, T.M. Pekarek, I. Miotkowski, A.K. Ramdas, Anisotropic magnetization of the III-VI diluted magnetic semiconductor $\operatorname{In}_{1-x} \mathrm{Mn}_{x} \mathrm{~S}$ in the mixed state // Phys. Rev. B, 72, 165201 (2005).

10. T.M. Pekarek, D.J. Arenas, I. Miotkowski, A.K. Ramdas, Magnetic and transport measurements on the layered III-VI diluted magnetic semiconductor $\operatorname{In}_{1-x} \mathrm{Mn}_{x} \mathrm{Se} / / \mathrm{J}$. Appl. Phys. 97, 10M106 (2005).

11. V.V. Slynko, O.G. Khandozhko, Z.D. Kovalyuk, A.V. Zaslonkin, V.E. Slynko, M. Arciszewska, W.D. Dobrowolski, Weak ferromagnetism in InSe:Mn layered crystals // Fizika tekhnika poluprovodn. 39, p. 806 (2005), in Russian.

12. V.V. Slyn'ko, A.G. Khandozhko, Z.D. Kovalyuk, V.E. Slyn'ko, A.V. Zaslonkin, M. Arciszewska, W. Dobrowolski, Ferromagnetic states in the $\mathrm{In}_{1-x} \mathrm{Mn}_{x} \mathrm{Se}$ layered crystals // Phys. Rev. B, 71, 245301 (2005).

13. T.M. Pekarek, L.H. Ranger, I. Miotkowski, A.K. Ramdas, Thermal hysteresis in the magnetization of the layered III-VI diluted magnetic semiconductor $\operatorname{In}_{1-x} \mathrm{Mn}_{x} \mathrm{Se} / / \mathrm{J}$. Appl. Phys. 99, 08D511 (2006).

14. G.V. Lashkarev, V.V. Slynko, Z.D. Kovalyuk, V.I. Sichkovskyi, M.V. Radchenko, P. Aleshkevych, R. Szymczak, W. Dobrowolski, R. Minikayev, Anomalies of magnetic properties of layered crystals InSe containing Mn // Mater. Sci. and Eng. C, 27, p. 1052 (2007).

15. G.V. Lashkarev, M.V. Radchenko, V.I. Sichkovskyi et al., Ferromagnetism of narrow-gap $\mathrm{Ge}_{1-x-y} \mathrm{Sn}_{\mathrm{x}} \mathrm{Mn}_{\mathrm{y}} \mathrm{Te}$ and layered $\mathrm{In}_{1-x} \mathrm{Mn}_{x}$ Se semiconductors // Acta phys. pol. A, 114, p. 1219-1227 (2008).

16. S. Popovic, A. Tonejc, B. Grzeta-Plenkovic, B. Celustka, R. Trojko, Revised and new crystal data for indium selenides // J. Appl. Crystallogr. 12(4), p. 416-420 (1979).

17. S.V. Vonsovskiy, Magnetism. Wiley, New York, 1974.

18. T. Dietl, A. Haury, Y. Merle d'Aubigne // Phys. Rev. B, 55, p. R3347 (1997).

19. J.B.C. Efrem D'Sa, P.A. Bhobe, K.R. Priolkar, A. Das, P.S.R. Krishna, P.R. Sarode, R.B. Prabhu, Low temperature magnetic structure of MnSe // Pramana - J. Phys. 63, p. 227-232 (2004).

20. R.M. Murray, B.C. Forbes, R.D. Heyding // Can. J. Chem. 50, p. 4059 (1972).

21. J.B.C. Efrem D'Sa, P.A. Bhobe, K.R. Priolkar, A. Das, S.K. Paranjpe, R.B. Prabhu, P.R. Sarode, Low temperature neutron diffraction study of $\mathrm{MnSe}$ // J. Magn. Magn. Mater. 285, p. 267-271 (2005).

22. V.A. Kul'bachinskii, Two-dimensional, Onedimensional, Zero-dimensional Structures and Sublattices. Moscow State University Publ., Moscow, 1998 (in Russian). 